\title{
The dynamics of E1A in regulating networks and canonical pathways in quiescent cells
}

\author{
Jean-Eudes Dazard ${ }^{1}$, Keman Zhang², Jingfeng Sha², Omar Yasin², Linda Cai ${ }^{2}$, Chien Nguyen², Mrinal Ghosh³, \\ Jennifer Bongorno ${ }^{4}$ and Marian L Harter ${ }^{2^{*}}$
}

\begin{abstract}
Background: Adenoviruses force quiescent cells to re-enter the cell cycle to replicate their DNA, and for the most part, this is accomplished after they express the E1A protein immediately after infection. In this context, E1A is believed to inactivate cellular proteins (e.g., p130) that are known to be involved in the silencing of E2F-dependent genes that are required for cell cycle entry. However, the potential perturbation of these types of genes by E1A relative to their functions in regulatory networks and canonical pathways remains poorly understood.
\end{abstract}

Findings: We have used DNA microarrays analyzed with Bayesian ANOVA for microarray (BAM) to assess changes in gene expression after E1A alone was introduced into quiescent cells from a regulated promoter. Approximately 2,401 genes were significantly modulated by E1A, and of these, 385 and 1033 met the criteria for generating networks and functional and canonical pathway analysis respectively, as determined by using Ingenuity Pathway Analysis software. After focusing on the highest-ranking cellular processes and regulatory networks that were responsive to E1A in quiescent cells, we observed that many of the up-regulated genes were associated with DNA replication, the cell cycle and cellular compromise. We also identified a cadre of up regulated genes with no previous connection to E1A; including genes that encode components of global DNA repair systems and DNA damage checkpoints. Among the down-regulated genes, we found that many were involved in cell signalling, cell movement, and cellular proliferation. Remarkably, a subset of these was also associated with p53-independent apoptosis, and the putative suppression of this pathway may be necessary in the viral life cycle until sufficient progeny have been produced.

Conclusions: These studies have identified for the first time a large number of genes that are relevant to E1A's activities in promoting quiescent cells to re-enter the cell cycle in order to create an optimum environment for adenoviral replication.

\section{Background}

Most somatic cells, including adult stem cells, are in a non-dividing or quiescent state (G0), and except for those that have become terminally differentiated or senescent, they can still re-enter the cell cycle when necessary. The molecular pathways that are responsible for maintaining cellular quiescence are largely unknown. However, it is known that these pathways can be influenced by external stimuli such as nutrients or growth factors, and that this in turn allows quiescent cells to grow, progress through $\mathrm{G1}$, and ultimately proliferate.

\footnotetext{
* Correspondence: mnh5@case.edu

${ }^{2}$ Department of Biochemistry, School of Medicine, Case Western Reserve

University, Cleveland, Ohio 44106, USA

Full list of author information is available at the end of the article
}

Human adenoviruses are another factor that can affect the pathways that control cellular quiescence [1]. These DNA viruses, which are a causative agent for various types of human diseases, typically infect non-dividing cells and force them into $S$ phase for replicating DNA. Ultimately, the viruses then use the cellular DNA precursors and the host enzymes to replicate their own DNA [2]. The first viral gene to be transcribed following adenovirus infection is E1A, and it encodes two major proteins of 289 (289R) and 243 amino acids (243R) [2]. The smaller size E1A is principally responsible for transitioning either human or rodent cells out of quiescence, and it can perform this function either alone or in the context of the virus [2-5]. This effect correlates with its ability to target key cellular proteins involved in regulating the cell
C Biomed Central 
cycle and chromatin function. Included in this group of proteins are the retinoblastoma family ( $\mathrm{pRb}, \mathrm{p} 107$, and p130), inhibitors of cyclin-dependent kinases, histone acetyltransferases, and other chromatin factors $[2,6,7]$.

The proteins $\mathrm{pRb}$ and $\mathrm{p} 130$ are especially important since they are both highly involved in regulating the E2F family of transcription factors (E2F1-E2F5). The E2F1-3a factors are activators of transcription and bind exclusively to $\mathrm{pRb}$, whereas p130 interacts specifically with E2F repressors such as E2F4 [8]. Studies have shown that the repressor complex p130-E2F4 associates with a substantial number of E2F-dependent genes in quiescent cells and that it serves to silence these genes by recruiting histone-modifying enzymes such as deacetylases (HDACs) and methyltransferases [9-11] to their respective promoters. The genes that are regulated by this complex include many that are involved in DNA replication, cell cycle control, and metabolism [11]. We have recently found that when expressed in quiescent cells, E1A can reverse the repression of at least two of these genes (e.g., $C D C 6$ and $C C N A$ ) by eliminating p130-E2F4 and HDAC complexes from their promoters, and then by recruiting a histone acetyltransferase to acetylate the surrounding nucleosomal histones $[4,5]$.

There are numerous E2F-dependent genes that are involved in a variety of biological processes [12], and when considering our previous work $[4,5]$, many of these have the potential to be targeted by E1A, leading to derepression of their expression in quiescent cells. E1A thus gives us an opportunity to identify, on a global scale, the gene regulatory networks that are required by the virus for its propagation in these cells. We have therefore used DNA microarray analyses with well-established statistical approaches on hybridized data from quiescent cells with or without the expression of E1A in order to address this important issue.

\section{Results and Discussion General Strategy}

Our previous studies have described a "Tet-on" inducible mouse cell line (Balb/c 3T3) that expresses the adenovirus E1A-243R protein in a regulated manner [4,5] An important feature of this cell line is that it can be brought to a state of quiescence by either contact inhibition or mitogen deprivation. Published data show that in this state, at least $95 \%$ of the cells are no longer in S phase, as measured by the absence of BrdU incorporation $[4,5]$. Once in this state, however, these cells can be made to transition into $\mathrm{S}$ phase by the induction of E1A following the addition of doxycycline (Dox), a tetracycline analog. In such an experiment, we typically find that $35-40 \%$ of the cells are incorporating BrdU after being treated with Dox for $12 \mathrm{hr}$, and $>98 \%$ of them express E1A [4]. Moreover, a microinjected E1A when compared to serum stimulation shortens the transition from $\mathrm{G} 0$ to $\mathrm{S}$ phase in quiescent cells with DNA synthesis beginning as early as $7 \mathrm{hr}$ after its expression [13]. Because our E1A-inducible cell line gives us the opportunity to identify E1A target genes in an unbiased and exclusive manner, we decided to use it in combination with DNA microarray analysis in order to identify, on a global scale E1A-mediated differentially expressed genes in quiescent cells.

\section{Statistical Approach}

Six independent cultures $(n=6)$ were used for cRNA labelling for the transcriptional profiling. The categorical factor under study consisted of a single 2-level Group Factor (GF): the control group, denoted 'Q' (quiescent cells without Dox, and therefore no E1A expression), versus the stimulated group, denoted 'S' (stimulated cells with Dox, and therefore with E1A expression). In this design, cell cultures were randomly assigned to treatments with three independent biological replicates per group. Therefore, this is an arrangement of treatments laid out on a balanced Complete Randomized Design (CRD) with no repeated measurements. In addition, no technical replicates were performed, no pooling was done, and there was no common reference sample.

One of the goals in high dimensional data mining is to identify which of the variables (such as mRNA and EST probes - sometimes abbreviated as genes) show evidence of differential expression between experimental conditions, while dealing with high dimensional contextual problems. To detect differential expression between experimental conditions when the number of variables greatly exceeds the number of observations or samples ( $p>>n$ paradigm), conventional regression techniques literally fall apart or are inappropriate at best. A standard approach in modelling high dimensional data is to fit the same statistical model individually to each outcome variable and test for the contrast or effect of interest using the hypothesis testing framework. Among the drawbacks of this univariate approach are the correlation structure (i.e. dependency) between the variables, which is totally ignored, while the risk of excessive conservativeness and of over fitting can be severely inflated [14].

In this study, we took advantage of the fact that the problem of differential expression can be cast into a variable selection problem in a regression setting. Recently, Bayesian model selection methods were proven the most reasonable approach to detect differentially expressed genes in high dimensional settings [15-17]. We employed one of these methods called Bayesian ANOVA (BAM). In effect, BAM is a model selection technique that relies on the so-called 'spike and slab' Bayesian hierarchical model used in parameter estimation. It is a special type of inferential regularization (i.e. borrowing information across genes), which builds a parsimonious model by selectively 
shrinking to zero only those (model) coefficients of genes that truly do not enter in the model [15]. This is an ideal property guaranteeing which genes will enter into the model and which will not, and allowing for optimal balancing of the number of false detections against false non-detections (i.e. false positive and false negative rates, or total gene misclassification errors), thereby leading to a more accurate and parsimonious model of truly differentially expressed genes [16,17].

Moreover, this technique is far superior to conventional one-at-a-time (univariate) hypothesis testing procedures, followed by multiple testing corrections that attempt to control only False Detection Rates (FDR) $[16,17]$. This is because controlling FDR tends to identify obviously varying genes but misses more subtle changes. This method also eliminates the problem of specifying arbitrarily False Discovery Rate $(F D R)$ cutoff values, and the drawback of excessive conservativeness [14]. In addition, BAM does not ignore the problem of dependency between the variables, which is frequently present within large datasets, and in biological data. Therefore, by using the BAM model, we were able to define a subset of differentially expressed genes of high statistical significance in response to E1A's expression in quiescent cells (see below).

\section{Microarray Analysis Identifies Genes that are modulated by E1A in Quiescent Cells}

To reveal differentially regulated genes in quiescent cells after E1A expression, we hybridized cRNA prepared from quiescent cells treated with or without Dox for 8 hr onto Agilent Whole Mouse Genome arrays, which allows for the interrogation of $\sim 25,000$ genes. This time point is approximately $1 \mathrm{hr}$ after the start of E1Amediated DNA synthesis in these cells, and therefore considers only genes that changed significantly in expression after cells transitioned from $\mathrm{G} 0$ into $\mathrm{S}$ phase.

To reduce the dimensionality of the problem (i.e., the number of potential probes at play on the microarray), we initially applied a pre-filtering step as described in Materials and Methods section, thereby reducing the number of probes to 10,632. After carrying out our Bayesian ANOVA on those 'Present' genes, we were able to select 2,401 genes on the arrays whose expression showed a significant change after quiescent cells had been treated with Dox [Figure 1 and Additional file 1: Supplemental Table S1]. Eight of these genes were unnamed, and 1,174 of the genes were up regulated, while 1,227 of them were down regulated. The total number of E1A-regulated genes is displayed in a Volcano and MA plot [Figure 2] as well as in a normalized quantile-quantile plot [Additional file 2: Supplemental Figure S1]. These plots importantly show (i) that only a few of these genes would have been discovered if only a conventional fold change analysis had been used [Figure 2 and Additional file 2: Supplemental Figure S1], and (ii) that conventional statistical approaches such as ANOVA or $t$-test would be inappropriate because of the violation of the normality assumption [Additional file 2: Supplemental Figure S1]. The top 50 genes that were up regulated or down regulated by this analysis are listed in Table 1. This analysis of E1A's activity in promoting quiescent cells into $\mathrm{S}$ phase has therefore allowed us to uncover, for the first time, significant factors to induce this event.

We next employed independent qRT-PCR assays to validate the array results for genes that displayed high or low fold change values, and which are known to have a direct or indirect role in DNA replication or cell cycle progression [Additional file 3: Supplemental Figure S2]. In the genes that were studied, and in the statistical methods that were employed, both of these technological platforms yielded changes that were proportionally conserved in their expression, indicating the reproducibility of change and the reliability of detection by microarray analysis.

These findings, along with our previous results [4,5], confirm that E1A alone has the capacity to affect $\sim 2,400$ genes in quiescent cells. As shown below, some of these genes are strictly related to maintaining cellular quiescence, while others are more important for helping cells to transition out of quiescence. It is worth noting that the number of genes $(\sim 6,400)$ whose promoters were reported to be bound by E1A in quiescent human cells, as determined by ChIP-on-chip analysis [18] is considerably larger than the number of genes found to be affected by E1A in our study. The basis for this difference is unknown, although the ChIP-on-chip analysis was performed on cells that had been infected with adenovirus for a period of $6 \mathrm{hr}$. In addition, the binding of E1A to promoters does not directly and immediately have to affect the expression of the respective genes. Another gene expression-profiling experiment using adenovirus infected quiescent human cells identified $\sim 2,100$ differentially expressed genes [1]. Interestingly, the earliest change in the concentrations of cellular RNA found in these cells was at $18 \mathrm{hr}$ post-infection, at least $6 \mathrm{hr}$ beyond the time when E1A was expressed [1]. Although E1A is likely involved in deregulating many of the cellular genes reported in the aforementioned studies, there is still the possibility of other viral proteins being contributors to this deregulation as well

\section{E1A Regulates a Variety of Biological Processes to Induce Cells out of Quiescence}

In order to identify the biological processes, canonical pathways and molecular networks that are potentially regulated by E1A for transitioning cells out of quiescence, 

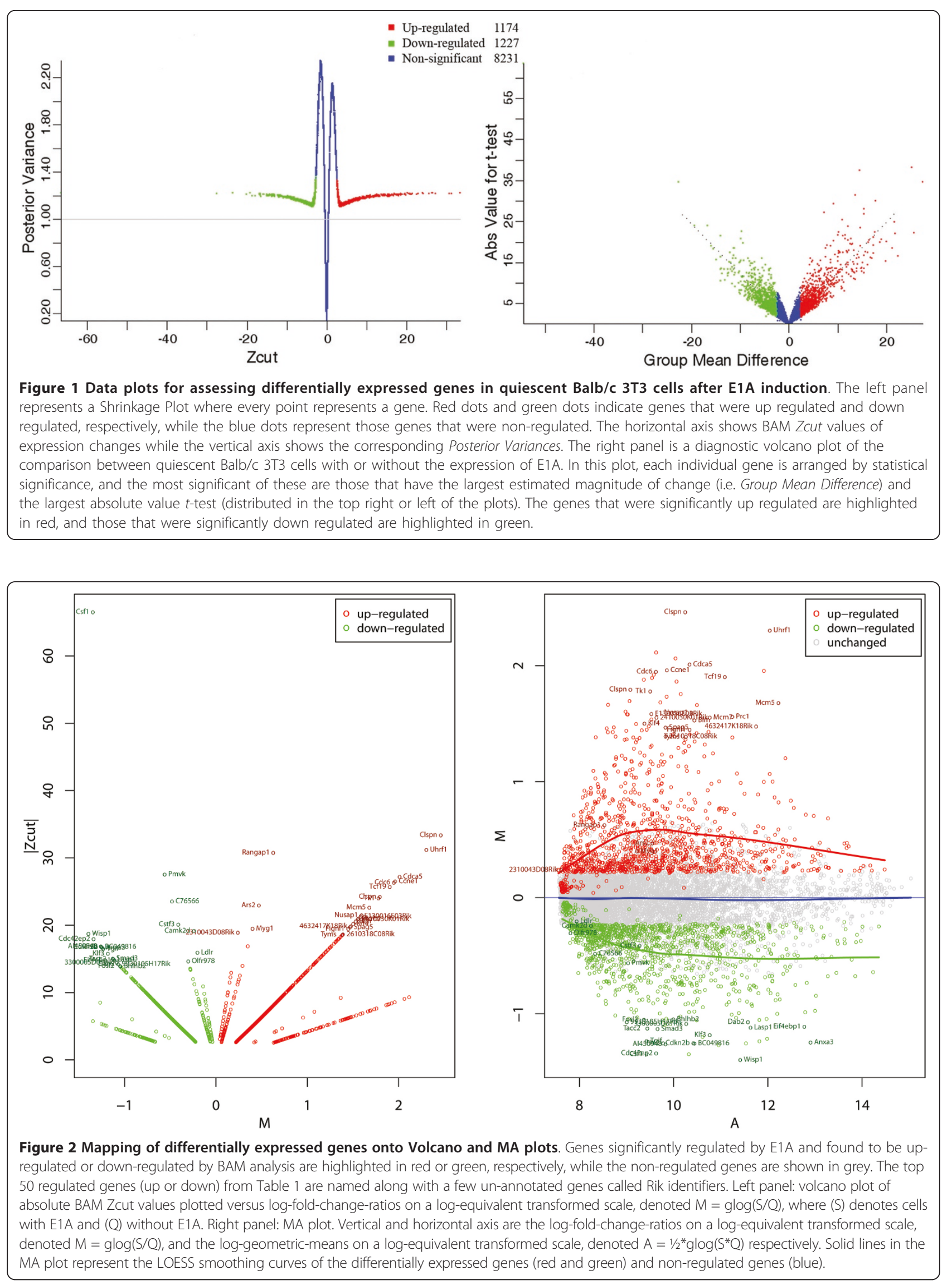
Table 1 List of top twenty up- and down-regulated genes found differentially expressed by BAM analysis in quiescent cells after E1A expression (Dox-treatment).

\begin{tabular}{|c|c|c|c|c|}
\hline Accession & Symbol & Definition & Zcut & $\operatorname{glog}(F C)$ \\
\hline \multicolumn{5}{|c|}{ Down-Regulated Genes } \\
\hline NM_007778.1 & Csf1 & Mus musculus colony stimulating factor 1 (macrophage) & -66.5094 & -1.348 \\
\hline NM_026784.1 & Pmvk & Mus musculus phosphomevalonate kinase & -27.5436 & -0.562 \\
\hline NM_178879.2 & C76566 & Mus musculus expressed sequence $\mathrm{C} 76566$ & -23.5382 & -0.482 \\
\hline NM_145529.1 & Cstf3 & Mus musculus cleavage stimulation factor, 3 pre-RNA, subunit 3 & -20.1076 & -0.413 \\
\hline NM_023813.2 & Camk2d & Mus musculus calcium/calmodulin-dependent protein kinase II, delta & -19.1488 & -0.242 \\
\hline NM_018865.1 & Wisp1 & Mus musculus WNT1 inducible signaling pathway protein 1 & -18.7272 & -1.396 \\
\hline NM_026772.1 & Cdc42ep2 & Mus musculus CDC42 effector protein (Rho GTPase binding) 2 & -17.9546 & -1.340 \\
\hline NM_001033476.1 & Al450948 & Mus musculus expressed sequence Al450948 & -16.851 & -1.261 \\
\hline NM_198612.1 & BC049816 & Mus musculus cDNA sequence BC049816 & -16.7728 & -1.255 \\
\hline NM_010212 & Fhl2 & Mus musculus four and a half LIM domains 2 & -16.7683 & -1.254 \\
\hline NM_007670.2 & Cdkn2b & Mus musculus cyclin-dependent kinase inhibitor 2B (p15) & -16.7461 & -1.253 \\
\hline NM_013470.1 & Anxa3 & Mus musculus annexin $\mathrm{A} 3$ & -16.6472 & -1.246 \\
\hline NM_009372.2 & Tgif & Mus musculus TG interacting factor & -16.4772 & -1.234 \\
\hline NM_010700.1 & Ldlr & Mus musculus low density lipoprotein receptor & -15.9324 & -0.203 \\
\hline NM_008453.2 & Klf3 & Mus musculus Kruppel-like factor 3 (basic) & -15.7751 & -1.183 \\
\hline NM_016769 & Smad3 & Mus musculus SMAD family member 3 & -15.0449 & -1.130 \\
\hline NM_206856.1 & Tacc2 & Mus musculus transforming, acidic coiled-coil containing protein 2 & -14.9903 & -1.126 \\
\hline NM_010688.2 & Lasp1 & Mus musculus LIM and SH3 protein 1 & -14.8866 & -1.119 \\
\hline NM_007918.2 & Eif4ebp1 & Mus musculus eukaryotic translation initiation factor 4E binding protein 1 & -14.7742 & -1.111 \\
\hline NM_147105.1 & Olfr978 & Mus musculus olfactory receptor 978 & -14.6434 & -0.304 \\
\hline XM_196166 & 3300005D01Rik & Mus musculus RIKEN cDNA 3300005D01 gene & -14.4319 & -1.086 \\
\hline NM_023118.1 & Dab2 & Mus musculus disabled homolog 2 (Drosophila) & -14.2169 & -1.071 \\
\hline \multicolumn{5}{|c|}{ Up-Regulated Genes } \\
\hline NM_175554.3 & Clspn & Mus musculus claspin homolog & 33.3736 & 1.378 \\
\hline NM_010931.2 & Uhrf1 & Mus musculus ubiquitin-like, containing PHD and RING finger domains, 1 & 31.1992 & 1.382 \\
\hline NM_011241 & Rangap1 & Mus musculus RAN GTPase activating protein 1 & 30.7663 & 1.384 \\
\hline NM_026410.1 & Cdca5 & Mus musculus cell division cycle associated 5 & 27.1852 & 1.387 \\
\hline NM_007633.1 & Cone1 & Mus musculus cyclin E1 & 26.512 & 1.390 \\
\hline NM_011799.1 & Cdc6 & Mus musculus cell division cycle 6 homolog (S. cerevisiae) & 26.3215 & 0.239 \\
\hline NM_025674.1 & Tcf19 & Mus musculus transcription factor 19 & 25.7018 & 1.449 \\
\hline NM_175554.3 & Clspn & Mus musculus claspin homolog & 24.2127 & 0.400 \\
\hline NM_009387 & Tk1 & Mus musculus thymidine kinase 1 & 23.9996 & 1.468 \\
\hline NM_031405.1 & Ars2 & Mus musculus arsenate resistance protein 2 & 22.9415 & 1.477 \\
\hline NM_008566.1 & Mcm5 & Mus musculus minichromosome maintenance deficient 5, cell division cycle 46 & 22.6281 & 1.502 \\
\hline NM_133851.1 & Nusap1 & Mus musculus nucleolar and spindle associated protein 1 & 21.4676 & 1.529 \\
\hline NM_001039556.1 & E130016E03Rik & Mus musculus RIKEN cDNA E130016E03 gene & 21.3155 & 1.552 \\
\hline NM_145150.1 & Prc1 & Mus musculus protein regulator of cytokinesis 1 & 20.9803 & 1.553 \\
\hline NM_008568.1 & Mcm7 & Mus musculus minichromosome maintenance deficient 7 (S. cerevisiae) & 20.8883 & 1.560 \\
\hline NM_026282.2 & 2410030K01Rik & Mus musculus RIKEN cDNA 2410030 K01 gene & 20.879 & 1.584 \\
\hline NM_007550 & $\mathrm{Blm}$ & Mus musculus Bloom syndrome homolog (human) & 20.5591 & 1.594 \\
\hline NM_008446.1 & Kif4 & Mus musculus kinesin family member 4 & 20.1831 & 1.679 \\
\hline NM_026640.1 & 4632417K18Rik & & 19.8413 & 0.470 \\
\hline NM_017407.1 & Spag5 & Mus musculus sperm associated antigen 5 & 19.7147 & 1.779 \\
\hline NM_021713.1 & Myg1 & Mus musculus melanocyte prolifeating gene 1 & 19.4864 & 1.794 \\
\hline NM_021891.2 & Fignl1 & Mus musculus fidgetin-like 1 & 19.4541 & 1.903 \\
\hline XM_130428.2 & 2310043D08Rik & & 18.9002 & 1.948 \\
\hline XM_149213.1 & 2610318C08Rik & Mus musculus RIKEN cDNA 2610318C08 gene & 18.6426 & 1.961 \\
\hline NM_021288.2 & Tyms & Mus musculus thymidylate synthase & 18.5966 & 2.010 \\
\hline NM_177733.2 & $\mathrm{E} 2 \mathrm{f} 2$ & Mus musculus E2F transcription factor 2 & 18.5614 & 0.627 \\
\hline
\end{tabular}


Table 1 List of top twenty up- and down-regulated genes found differentially expressed by BAM analysis in quiescent cells after E1A expression (Dox-treatment). (Continued)

\begin{tabular}{llll}
\hline NM_007891.1 & E2f1 & Mus musculus E2F transcription factor 1 & 18.5401 \\
NM_011284.2 & Rpa2 & Mus musculus replication protein A2 & 2.303 \\
\hline
\end{tabular}

Transcripts are ranked by statistical significance (Zcut values) from top to bottom. Direction of change is indicated by the Zcut sign. Generalized-log-fold change values $(g \log (F C))$ represent the corresponding magnitude of regulation of the selected genes.

we up-loaded the BAM Zcut values of the E1A-modulated genes into the Ingenuity Pathway (IPA) software. We applied specific filters (see Materials and Methods) for limiting our experimental observations to information that would be most relevant to the reversibility of quiescent cells, and which left 385 up/down-regulated molecules/ genes that were eligible for generating networks, and 1033 molecules/genes, which were eligible for functional and canonical pathway analysis. Of interest is that regardless of whether the IPA filter for mouse or human was used, the results from the respective analyses were strikingly similar.

After performing an IPA core analysis, we found that the E1A-243R was able to modulate sets of genes that are known to function in several key cellular processes, and which were either upregulated or downregulated in their expression (Figure 3). In particular, genes involved in transitioning cells out of quiescence (e.g., CDK2,
E2F1, E2F2, RBL1, RB1, ID2, SKP2, BRAC2, TP53, $C C N E 1, C D C 25 C$, and $A T M)$ and for making chromatin competent for DNA replication (e.g., CDC6, CDT1, the MCM replicative helicase complex ( $M c m 2-M c m 7$, $O R C 1 L$, and $O R C 6 L$ ) were significantly up-regulated after E1A was expressed in quiescent cells [Additional file 1: Supplemental Table S1]. We also identified several other genes which were significantly up regulated and are known to be required for the metabolism and synthesis of DNA [19]. These included POLD1, POLA2, FEN1, KIN, PRIM1, PRIM2, HELB, NASP, and LIG1. Notably, the promoter regions of most of these genes are known to be occupied by the E2F4 repressor complex in quiescent cells [20]. Given that, E1A alone can dissociate this transcriptional repressor from an inactive endogenous CDC6 promoter in quiescent cells $[4,5]$, this new data suggests that E1A may also have the

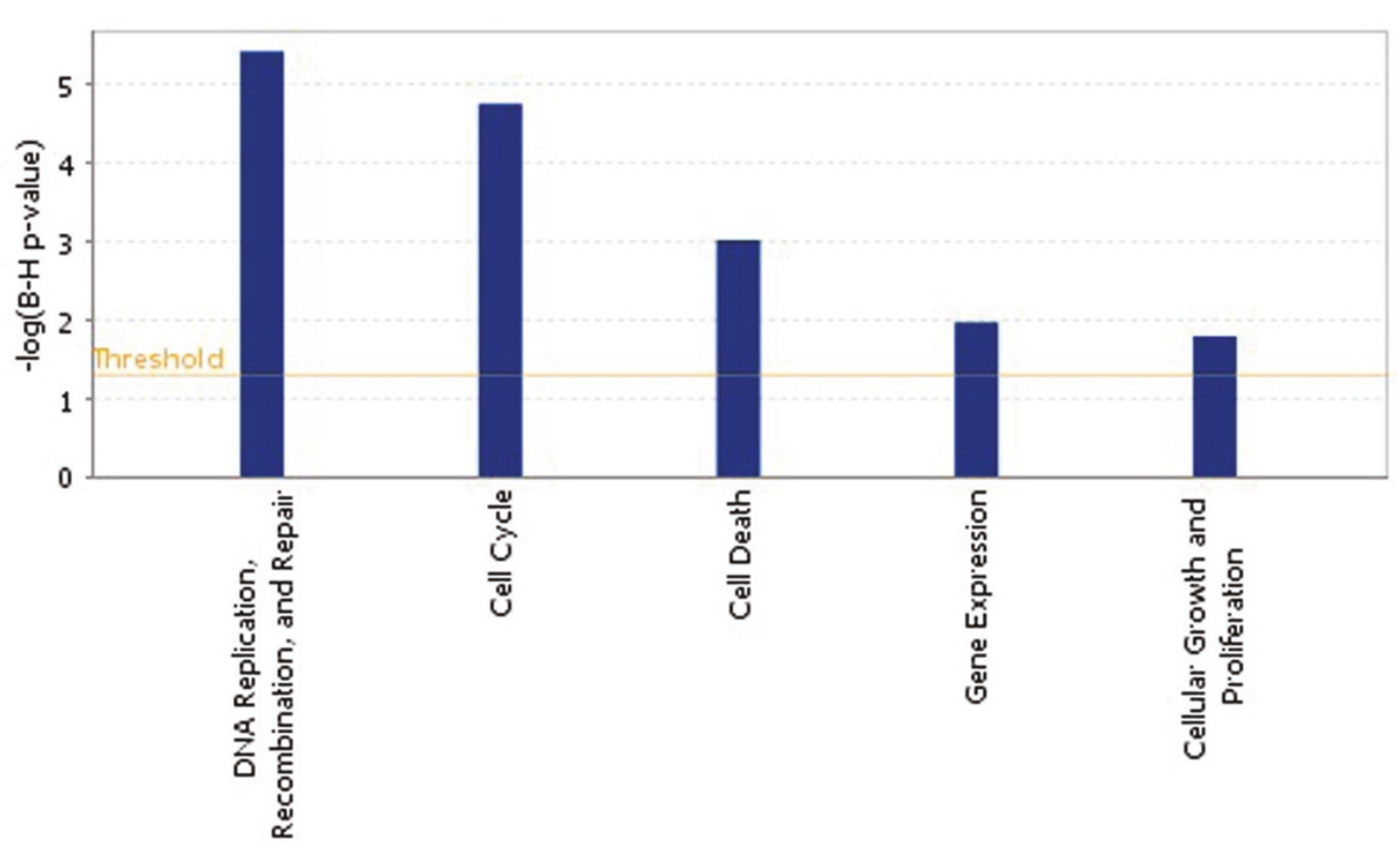

Figure 3 Molecular and cellular functions most significantly regulated by E1A expression in quiescent cells. The Benjamini-Hochberg (BH) method was used to adjust the right-tailed Fisher's Exact $t$-test $p$-values, which measure how significant each pathway is. Functions having the highest $p$-values are shown. For a complete listing of the genes used in this analysis, see Supplemental Table S1 [Additional file 1: Supplemental Table S1]. 
ability to directly affect the promoters of other genes that are subject to E2F4 repression in these cells. In effect, this may be one way in which E1A contributes to the growth of viruses in the infected cells.

Although there is evidence to suggest that quiescent mouse cells lack global genomic DNA repair, they do exhibit proficient transcription-coupled DNA repair $[21,22]$. Interestingly, the IPA analysis identified clusters of up regulated genes that are involved in different DNA repair pathways, as well as in checkpoint controls, and the DNA damage response (DDR) (Table 2). As noted in the Table, a majority of the genes can bind E2F4 [12], and at least one-third of them have been found to be associated with this factor in quiescent cells [20], and therefore have the potential of being up regulated by E1A.

It is interesting to note that all of the DNA repair genes that are up regulated in response to E1A expression in quiescent cells fall into three categories, mismatch and excision repair (e.g., $M S H 2, M S H 6, M L H 1, C D C 7$, and $P M S 2)$, and DNA double-strand break repair by homologous recombination (e.g., ATM, BRCA1, BRCA2, MRE11, and LIG1) or non-homologous end-joining (e.g., $A T M$, NBS1, MRE11, and XRCC1and 6). This is underscored and graphically illustrated by additional IPA analyses (Figure 4A and Figure 4B), which identified each of these DNA repair systems ( $p$ values 7.76E-07, 1.14E-04, and 1.83-02, respectively) as significant categories within Canonical Pathways. The necessity of these DNA repair systems during E1A-mediated transition out of quiescence and into $\mathrm{S}$ phase, either alone or in the context of virus is unclear. However, it is conceivable that the rapid and abnormal progression into $\mathrm{S}$ phase induced by E1A could lead to DNA damage (single-stranded or doublestranded), possibly generated by an instability in the replication forks (e.g., stopping or pausing because of the depletion of the nucleotide pool) as they move away from their respective origins [19]. Alternatively, the restoration of global DNA repair systems following the E1Amediated re-entry of quiescent cells into the cell cycle could be a natural phenomenon so that the proliferating cells can cope with various kinds of DNA damage, if need be.

Finally, additional analysis by the IPA software revealed a set of genes that were significantly down regulated in the E1A expressing cells [Additional file 1: Supplemental Table S1]. Genes of interest that were affected in the functional category of Cellular Growth and Proliferation included the cyclin dependent kinases inhibitors CDKN2A (p16) and CDKN2B (p15) as well as $P T K 2$, SOCS, TOB1, and CAV1, which has been suggested to play an important role in maintaining cells in a quiescent state [23].

\section{E1A Regulates Specific Cellular Networks and Canonical Pathways in Quiescent Cells}

To determine whether any of the differentially expressed genes in the E1A expressing cells are interacting with one another, we used the IPA software to generate a listing of gene regulatory networks. Our first analysis was done on the up-regulated genes, and this computation produced one top network that was essentially associated with DNA replication, DNA repair, and the re-entry of cells into the cell cycle. As illustrated in Figure 5, many of the up-regulated genes in this system are involved in DNA replication ( $p$-value 9.36E-15). For example, genes encoding the activating E2F transcriptional factors (E2F1 and $E 2 F 2$ ), as well as some of the more important components of DNA replication (e.g., CDC6, MCMs, CCNE1, $P C N A$, and $C D K 2$ ) were found to have positions in this network. Genes included in the categories of DNA repair and the response to DNA damage were $M C M 7, M E N 1$, MSH2, TP53, ATM, PCNA TYMS, RRM2, and MCPH1. It should be noted that $M C M 7$, in addition to its role in the replication of DNA, might also be involved in the transmission of DNA damage signals [24]. Clearly, this regulatory network not only highlights the interactions between genes that are active in DNA replication but also those that function in DNA repair and DDR. This is consistent with the canonical pathway displayed in Figure 4B. Finally, this network includes $H D A C 1, E E D$, and $E Z H 2$, which are E2F-dependent genes that participate in the methylation of lysine 27 on histone H3 (H3K27), a marking that is largely associated with the repression of genes [25]. Indeed, a gain in the di- or tri-methylation of H3K27 at specific sites on chromatin might account for at least some of the genes that were down regulated in quiescent cells after E1A expression (Table 1).

Coordinated interactions between the down-regulated genes in the E1A-modulated dataset were also revealed by using the IPA software. Two of the regulatory networks that were identified by this analysis are presented in Figure 6. Not surprisingly, the genes comprising the first network were linked to biological processes such as cell-to-cell signalling ( $p$-value of 6.34E-13), cellular development ( $p$-value of 2.57E-12), and cellular growth and proliferation ( $p$-value of $6.43 \mathrm{E}-10)$. The transcriptional and translational co-repressors SAP3O and EIF4EBP1 play an important role in this network as does the SOCS2, SOCS3 and EGFR, which are known to increase the differentiation of cells. Of particular interest is that this network also contained the Toll-like receptor proteins TLR4 and TLR6, which were interconnected with the two signalling pathways (Figure 6). Both of these proteins play a fundamental role in pathogen recognition and the activation of innate immunity [26]. Therefore, their E1A-mediated repression in quiescent 
Table 2 Identification of up regulated genes identified in E1A expressing quiescent cells and required for DNA repair, checkpoint controls, and the DNA damage response.

\begin{tabular}{|c|c|c|c|c|c|}
\hline Accession & $\begin{array}{l}\text { Gene } \\
\text { Symbol }\end{array}$ & Description & $\begin{array}{l}\text { E2F4 } \\
\text { binding }\end{array}$ & Zcut & $\begin{array}{r}g l o g \\
(F C) \\
\end{array}$ \\
\hline \multicolumn{6}{|c|}{$\begin{array}{c}\text { DNA repair processes: homologous recombination, mismatch/excision repair, } \\
\text { and non-homologous end-joining }\end{array}$} \\
\hline NM_007499.1 & ATM & Ataxia telangiectasia mutated & + & 7.498 & 0.585 \\
\hline NM_007550 & BLM & Bloom syndrome, RecQ helicase-like & + & 20.559 & 1.529 \\
\hline NM_009764.2 & BRCA1 & Breast cancer 1 , early onset & - & 4.757 & 0.381 \\
\hline NM_009765.1 & BRCA2 & Breast cancer 2, early onset & - & 12.234 & 0.927 \\
\hline $\begin{array}{l}\text { NM_007691.2 } \\
\text { NM_009863.1 }\end{array}$ & $\begin{array}{l}\text { CHEK1 } \\
\text { CDC7 }\end{array}$ & $\begin{array}{c}\text { CHK1 checkpoint homolog (S. Pombe) Cell division cycle } 7 \text { homolog (S. } \\
\text { cerevisiae) }\end{array}$ & ++ & $\begin{array}{r}3.202 \\
17.694\end{array}$ & $\begin{array}{l}0.261 \\
1.321\end{array}$ \\
\hline NM_028119.2 & DDB2 & Damage-specific DNA binding protein 2, $48 \mathrm{kDa}$ & + & 9.204 & 0.709 \\
\hline NM_177752.2 & EME1 & Essential meiotic endonuclease 1 homolog 1 (S. pombe) & - & 2.820 & 0.232 \\
\hline NM_001033244.1 & FANCD2 & Fanconi anemia, complementation group D2 & + & 9.508 & 0.730 \\
\hline NM_007999.2 & FEN1 & Flap structure-specific endonucleae 1 & + & 8.577 & 1.957 \\
\hline NM_010436.2 & $\mathrm{H} 2 \mathrm{AFX}$ & $\mathrm{H} 2 \mathrm{~A}$ histone family, member $\mathrm{X}$ & + & 4.634 & 1.095 \\
\hline NM_010715.1 & LIG1 & Ligase 1, DNA, ATP-dependent & + & 18.060 & 1.348 \\
\hline NM_026810.1 & MLH1 & MutL homolog 1, colon cancer, nonpolyposis type 2 (E. coli) & + & 2.794 & 0.230 \\
\hline NM_018736.2 & MRE11A & MRE11 meiotic recombination 11 homolog A (S. cerevisiae) & - & 5.432 & 0.432 \\
\hline NM_008628.1 & $\mathrm{MSH} 2$ & MutS homolog 2, colon cancer, nonpolyposis type 1 (E. coli) & + & 3.107 & 0.254 \\
\hline NM_010830.1 & MSH6 & MutS homolog 6 (E.coli) & - & 13.241 & 1.000 \\
\hline NM_013752 & NBN & Nibrin & - & 8.326 & 0.645 \\
\hline NM_009632.2 & PARP2 & Poly (ADP-ribose) polymerase 2 & + & 7.656 & 0.596 \\
\hline NM_011045.1 & PCNA & Proliferating cell nuclear antigen & + & 10.999 & 0.838 \\
\hline NM_008886.1 & PMS2 & PMS2 postmeiotic segregation increased 2 (S. cerevisiae) & - & 8.409 & 0.651 \\
\hline NM_011131.2 & POLD1 & Polymerase (DNA directed) delta 1, catalytic subunit $125 \mathrm{kDa}$ & + & 7.744 & 0.603 \\
\hline $\begin{array}{l}\text { NM_030715.2 } \\
\text { NM_008949.2 }\end{array}$ & $\begin{array}{l}\text { POLH } \\
\text { PSMC3IP }\end{array}$ & Polymerase (DNA directed), eta PSMC3 interacting protein & -+ & $\begin{array}{r}3.448 \\
17.496\end{array}$ & $\begin{array}{l}0.825 \\
1.307\end{array}$ \\
\hline $\begin{array}{l}\text { NM_013917.1 } \\
\text { NM_021385.1 }\end{array}$ & $\begin{array}{l}\text { PTTG1 } \\
\text { RAD18 }\end{array}$ & Pituitary tumor-transforming 1 RAD18 homolog (S. cerevisiae) & ++ & $\begin{array}{l}7.190 \\
7.629\end{array}$ & 0.562 \\
\hline NM_009012.1 & RAD50 & RAD50 homolog (S. cerevisiae) & + & 4.064 & 0.328 \\
\hline NM_011234.2 & RAD51 & RAD51 homolog (RecA homolog, E. coli) (S. cerevisiae) & + & 12.514 & 0.947 \\
\hline NM_009013.1 & RAD51AP1 & RAD51 associated protein 1 & + & 9.638 & 0.740 \\
\hline NM_009015.2 & RAD54L & RAD54-like (S. cerevisiae) & + & 9.320 & 2.115 \\
\hline NM_026653.1 & RPA1 & Replication protein $\mathrm{A} 1,70 \mathrm{kDa}$ & - & 9.177 & 0.707 \\
\hline NM_011237.1 & RAD9A & RAD9A homolog A & - & 4.501 & 0.361 \\
\hline NM_023042.1 & RECQL & RecQ protein-like (DNA helicase Q1-like) & + & 5.724 & 0.125 \\
\hline NM_021419.1 & RNF8 & Ringfinger protein 8 & + & 2.663 & 0.222 \\
\hline NM_011677.1 & UNG & Uracil-DNA glycosylase & + & 4.901 & 0.475 \\
\hline $\begin{array}{l}\text { NM_133786.3 } \\
\text { NM_153808.1 }\end{array}$ & $\begin{array}{l}\text { SMC4L1 } \\
\text { SMC5L1 }\end{array}$ & $\begin{array}{r}\begin{array}{r}\text { Structural maintenance of chromosomes four-like } 1 \text { Structural maintenance of } \\
\text { chromosomes five-like } 1\end{array}\end{array}$ & -- & $\begin{array}{r}4.290 \\
10.047\end{array}$ & $\begin{array}{l}0.345 \\
0.769\end{array}$ \\
\hline $\begin{array}{l}\text { XM_127444.3 } \\
\text { NM_010247.1 }\end{array}$ & $\begin{array}{l}\text { TRIP13 } \\
\text { XRCC6 }\end{array}$ & $\begin{array}{c}\text { Thyroid hormone receptor interactor } 13 \text { X-ray repair complementing defective } \\
\text { repair in Chinese hamster }\end{array}$ & +- & $\begin{array}{l}3.630 \\
3.039\end{array}$ & $\begin{array}{l}0.294 \\
0.732\end{array}$ \\
\hline Accession & $\begin{array}{l}\text { Gene } \\
\text { Symbol }\end{array}$ & Description & $\begin{array}{l}\text { E2F4 } \\
\text { binding }\end{array}$ & Zcut & $\begin{array}{r}g l o g \\
(F C)\end{array}$ \\
\hline \multicolumn{6}{|c|}{ DNA damage response } \\
\hline NM_007499.1 & ATM & Ataxia telangiectasia mutated & + & 7.498 & 0.585 \\
\hline NM_009764.2 & BRCA1 & Breast cancer 1 , early onset & - & 4.757 & 0.381 \\
\hline NM_009765.1 & BRCA2 & Breast cancer 2 , early onset & - & 12.234 & 0.927 \\
\hline NM_007691.2 & CHEK1 & CHK1 checkpoint homolog (S. Pombe) & + & 3.202 & 0.261 \\
\hline NM_001033244.1 & FANCD2 & Fanconi anemia, complementation group D2 & + & 9.508 & 0.730 \\
\hline NM_008316.2 & HUS1 & HUS1 checkpoint homolog (S. pombe) & - & 4.438 & 0.357 \\
\hline NM_010774.1 & MBD4 & Methyl-CpG binding domain protein 4 & - & 8.816 & 0.681 \\
\hline
\end{tabular}


Table 2 Identification of up regulated genes identified in E1A expressing quiescent cells and required for DNA repair, checkpoint controls, and the DNA damage response. (Continued)

\begin{tabular}{|c|c|c|c|c|c|}
\hline NM_008583.1 & MEN1 & Multiple endocrine neoplasia 1 & $?$ & 4.995 & 0.399 \\
\hline NM_026810.1 & MLH1 & MutL homolog 1, colon cancer, nonpolyposis type 2 (E. coli) & + & 2.794 & 0.230 \\
\hline NM_008628.1 & $\mathrm{MSH} 2$ & MutS homolog 2, colon cancer, nonpolyposis type 1 (E. coli) & + & 3.107 & 0.254 \\
\hline NM_010830.1 & MSH6 & Muts homolog 6 (E. coli) & - & 13.241 & 1.000 \\
\hline NM_008884.2 & PML & Promyelocytic leukemia & - & 3.155 & 0.257 \\
\hline NM_011237.1 & RAD9A & RAD9 homolog A (S. pombe) & - & 4.501 & 0.361 \\
\hline NM_011234.2 & RAD51 & RAD51 homolog (RecA homolog, E. coli) (S. cerevisiae) & + & 12.514 & 0.947 \\
\hline NM_009015.2 & RAD54L & RAD54-like (S. cerevisiae) & + & 9.320 & 2.115 \\
\hline NM_026653.1 & RPA1 & Replicaton protein A1, $70 \mathrm{kDa}$ & + & 9.177 & 0.707 \\
\hline NM_011623.1 & TOP2A & Topoisomerase (DNA) 11 alpha $170 \mathrm{kDa}$ & + & 8.224 & 1.880 \\
\hline NM_011640.1 & TP53 & Tumor protein p53 & - & 6.333 & 0.499 \\
\hline NM_010247.1 & XRCC6 & X-ray repair complementing defective repair in Chinese hamster & - & 3.039 & 0.732 \\
\hline Accession & $\begin{array}{l}\text { Gene } \\
\text { Symbol }\end{array}$ & Description & $\begin{array}{l}\text { E2F4 } \\
\text { binding }\end{array}$ & Zcut & $\begin{array}{l}\text { glog } \\
(F C)\end{array}$ \\
\hline \multicolumn{6}{|c|}{ DNA replication checkpoints and checkpoint control } \\
\hline NM_007499.1 & ATM & Ataxia telangiectasia mutated & + & 7.498 & 0.585 \\
\hline NM_026014.3 & CDT1 & Chromatin licensing and DNA replication factor 1 & - & 4.137 & 0.983 \\
\hline $\begin{array}{l}\text { NM_007691.2 } \\
\text { NM_008316.2 }\end{array}$ & $\begin{array}{l}\text { CHEK1 } \\
\text { HUS1 }\end{array}$ & CHK1 checkpoint homolog (S. Pombe) HUS1, checkpoint homolog (S. pombe) & ++ & $\begin{array}{l}3.202 \\
4.438\end{array}$ & $\begin{array}{l}0.261 \\
0.357\end{array}$ \\
\hline NM_008583.1 & MEN1 & Multiple endocrine neoplasia 1 & - & 4.995 & 0.399 \\
\hline NM_008628.1 & MSH2 & MutS homolog 2, colon cancer, nonpolyposis type 1 (E. coli) & + & 3.107 & 0.254 \\
\hline NM_134092.2 & MTBP & Mdm2, transformed 3T3 cell double minute 2 & + & 9.084 & 0.700 \\
\hline NM_011237.1 & RAD9A & RAD9 homolog A (S. pombe) & + & 4.501 & 0.361 \\
\hline NM_011640.1 & TP53 & Tumor protein p53 & - & 6.333 & 0.499 \\
\hline
\end{tabular}

1,2 Data for the binding of E2F4 was obtained from Ren et al. 2002 (superscript 1) and Xu et al. 2007 (superscript 2). Generalized-log-fold change values (glog(FC)) represent the corresponding magnitude of regulation of the selected genes.

cells is provocative since this may represent an additional way of neutralizing the host's antiviral defence. Indeed, it is well established that adenoviruses encode other proteins that function to inhibit host immune responses, for example, killing by $\mathrm{CD}^{+}$cytotoxic $\mathrm{T}$ lymphocytes [27].

The second network displayed in Figure 7A contains down-regulated genes that are primarily involved in apoptosis ( $p$-value of 1.02E-17) and developmental processes ( $p$-value of 5.46E-16). This network includes a number of pro-apoptotic genes (e.g., $B A X$ and $B I D$ ) whose mRNAs decreased in response to E1A expression. The change in expression of these genes and their interactions in apoptotic pathways were also made clear after the down-regulated genes in the database were subjected to canonical pathway analysis. Among the pathways that were revealed in this study, apoptotic signalling ( $p$-value of 9.81E-9), was one of the most significant to be perturbed by E1A expression. This suggests that E1A may be blocking p53-independent apoptosis when it is expressed in quiescent cells (Figure 7B). In effect, E1A may be temporarily suppressing the p53independent apoptosis that has been shown to be a consequence of adenovirus infection, and which is apparently mediated by the larger size E1A protein
(289R) and the adenovirus product E4orf4 [28]. Notably, it has been suggested that apoptosis may be promoted by adenovirus to kill cells at the very end of its replicative cycle [28]. This may explain why the virus has developed additional mechanisms, for example the encoding of the E1B-55K and $-19 \mathrm{~K}$ proteins, for controlling apoptosis during the infection.

\section{Conclusions}

One of the most important conclusions to be drawn from the study is the ability of the small size E1A protein of 243R to perturb a rather large number of cellular processes in quiescent cells. This is most likely due to its ability to re-organize chromatin structure $[4,5]$, an effect which in turn leads to the reprogramming of 2400 cellular genes. According to IPA analysis, 271 of these differentially expressed genes are significantly associated with DNA replication, DNA repair, the cell cycle and cell death, all of which are necessary for efficient viral replication. This analysis also revealed perturbation in other cellular processes, including cell-to-cell signalling, cellular development and cellular growth, which may be required to provide an optimal environment for the replication of the virus. Finally, our studies suggest a role for E1A in altering metabolic homeostasis 


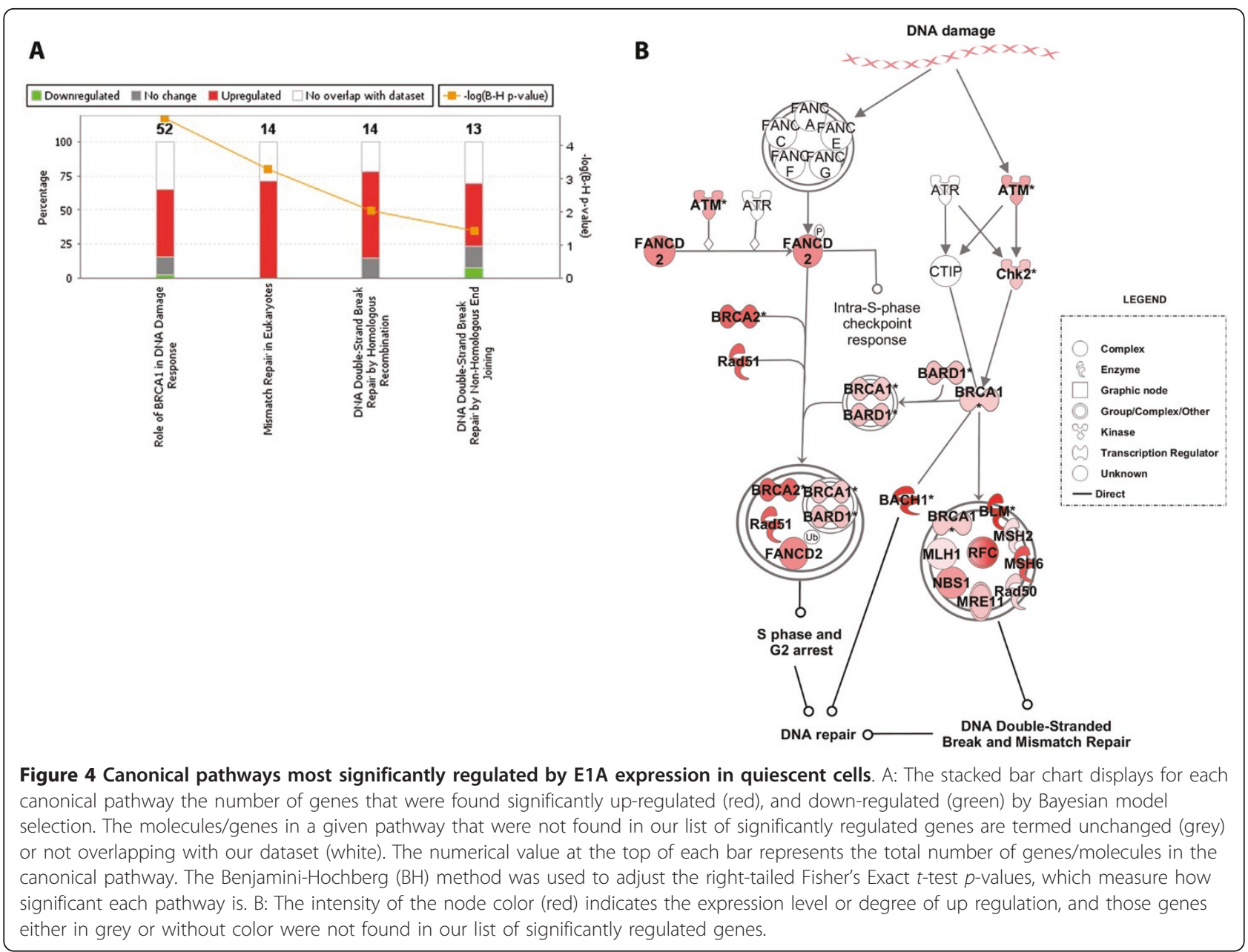

in quiescent cells [Additional file 4: Supplemental Figure S3]. Therefore, a more complete understanding of whether these metabolic differences are necessary for viral growth will be an important goal for future studies.

\section{Methods}

\section{Cell Culture}

The Tet-on inducible Balb/c 3T3 cell line expressing a wild-type 243 amino-acid E1A protein has been previously described $[5,29]$. This particular clone, which is maintained in DMEM supplemented with antibiotics, Lglutamine, blasticidin/zeocin, and 10\% fetal bovine serum (Tet system approved; Clontech), expresses a modest amount of E1A after the addition of $100 \mathrm{ng} / \mathrm{ml}$ of doxycycline (Dox), and this expression was sustained [Additional file 5: Supplemental Figure S4] after the cells had been subcultured three different times in preparation for the extraction of RNA and subsequent DNA microarray analysis (see below). After reaching a confluency of $40-60 \%$, the cells in the absence of Dox were rendered quiescent by shifting them to medium containing $0.05 \%$ serum for a period of $60 \mathrm{hr}$, as previously described $[4,5]$. For DNA microarray analysis, quiescent cells in $0.05 \%$ medium for a period of $60 \mathrm{hr}$ were treated with a $100 \mathrm{ng} / \mathrm{ml}$ of Dox for $8 \mathrm{hr}$ and then harvested for the extraction of RNA.

\section{Isolation of RNA and Microarray Analysis}

Total RNA was isolated by adding Trizol reagent (Invitrogen) directly to the monolayer of cells, and according to the manufacturer's instructions. The isolated RNA was then subjected to the RNeasy MinElute Cleanup Kit to ensure high-quality RNA, and afterwards, the RNA sample was subjected to a DNAse I treatment (DNAfree, Ambion) to remove all traces of contaminating DNA. The quantity and the quality of the RNA was assessed by NanoDrop 1000 (Thermo Scientific, Waltham, MA) and by the $18 \mathrm{~S} / 28 \mathrm{~S}$ ribosomal peak intensity on an Agilent Bioanalyzer.

The microarray analysis was performed at the Cleveland Clinic Genomics core facility by using Agilent Mouse Whole Genome Arrays (Agilent, Santa Clara, 


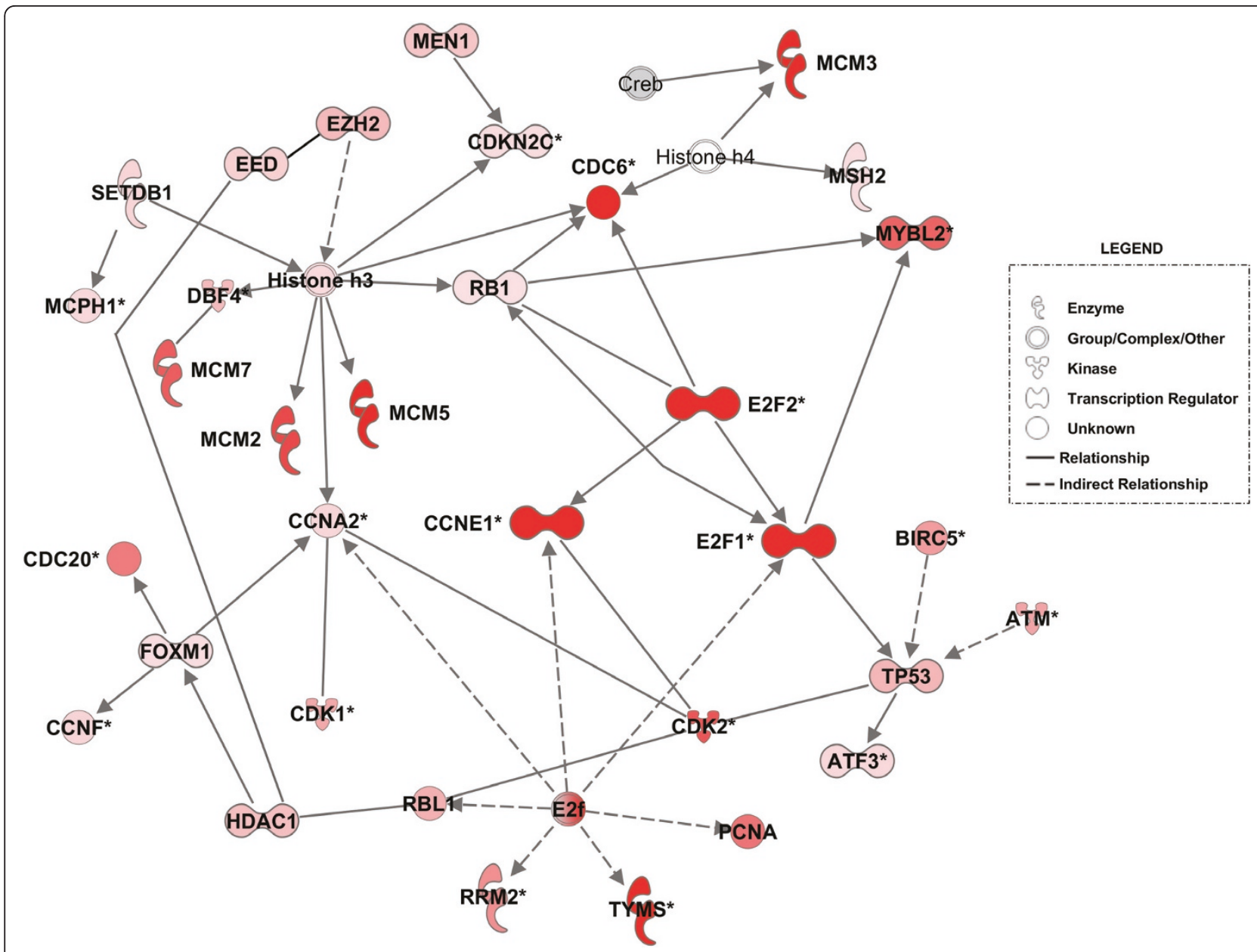

Figure 5 A network of up-regulated genes in quiescent cells after E1A expression. The intensity of the node color (red) indicates the expression level or degree of up-regulation. The gene represented in grey (CREB) and the ones without color were not found in our list of significantly regulated genes. Both direct (solid line) and indirect (broken line) relationships between the genes are indicated. Cellular functions most significant to the genes in this network included cell cycle re-entry and DNA replication or repair.

CA) in a balanced block design. Total RNA (250 ng) was reverse transcribed into cRNA and biotin-UTP labeled using the Illumina Total Prep RNA Amplification kit (Ambion). cRNA was quantified using the NanoDrop 1000 spectrophotometer and its quality (size distribution) was further analyzed on a $1 \%$ agarose gel. Afterwards, the cRNA was hybridized to high-density oligonucleotide density arrays (an Illumina MouseRef8v1.1 Expression BeadChip) containing 24, 613 probes or Target IDs, as well as ESTs sequences. The arrays were then washed, accordingly, and afterwards scanned using an Illumina BeadArray Reader.

\section{qRT-PCR Analysis}

The genes showing p-values less than 0.05 , and a change in gene expression of more than 2-fold when compared to control (determined by BeadStudio software) were selected for qRT-PCR analysis in order to validate the microarrays.
The RNA used for qRT-PCR was isolated as described above for the microarray studies. cDNA was synthesized by using Invitrogen's SuperScript III First-Strand Synthesis kit with random hexamer primers. The resultant samples were then mixed with $\mathrm{iQ}^{\mathrm{TM}} \mathrm{SYBR}^{\circledR}$ Green Supermix (BioRad) and with specific primer sets purchased from Invitrogen and designed by programs, which included NCBI-BLAST, USCS Genome Browser, and Primer3. Primer pairs for the respective mRNAs under study were: Mcm3 (forward), 5'-TGACCTGCTCTTCATCATGC-3'; Mcm3 (reverse), 5'-CTGTG GCCCAGGATATC CACT3'; Cdc6 (forward), 5'-AGGAGCCAGACAGTC CTCAA3', Cdc6 (reverse), 5'-GGGTCAAAAGCAGCAAAGAG; Cyclin E (forward), 5'-CACAACATCCGACC CACAC-3', Cyclin E (reverse) 5'-GGCAG GTTGGTCATTCTGT-3'; Csf1 (forward), 5'-ACAACACCCCCAATGCTAAC-3', Csf1(reverse), 5'-ATGGAAAGTTCGGACAC AGG-3'; E2F1 (forward), 5'-GATCGAAGCTTTAATGGAGCG-3', 


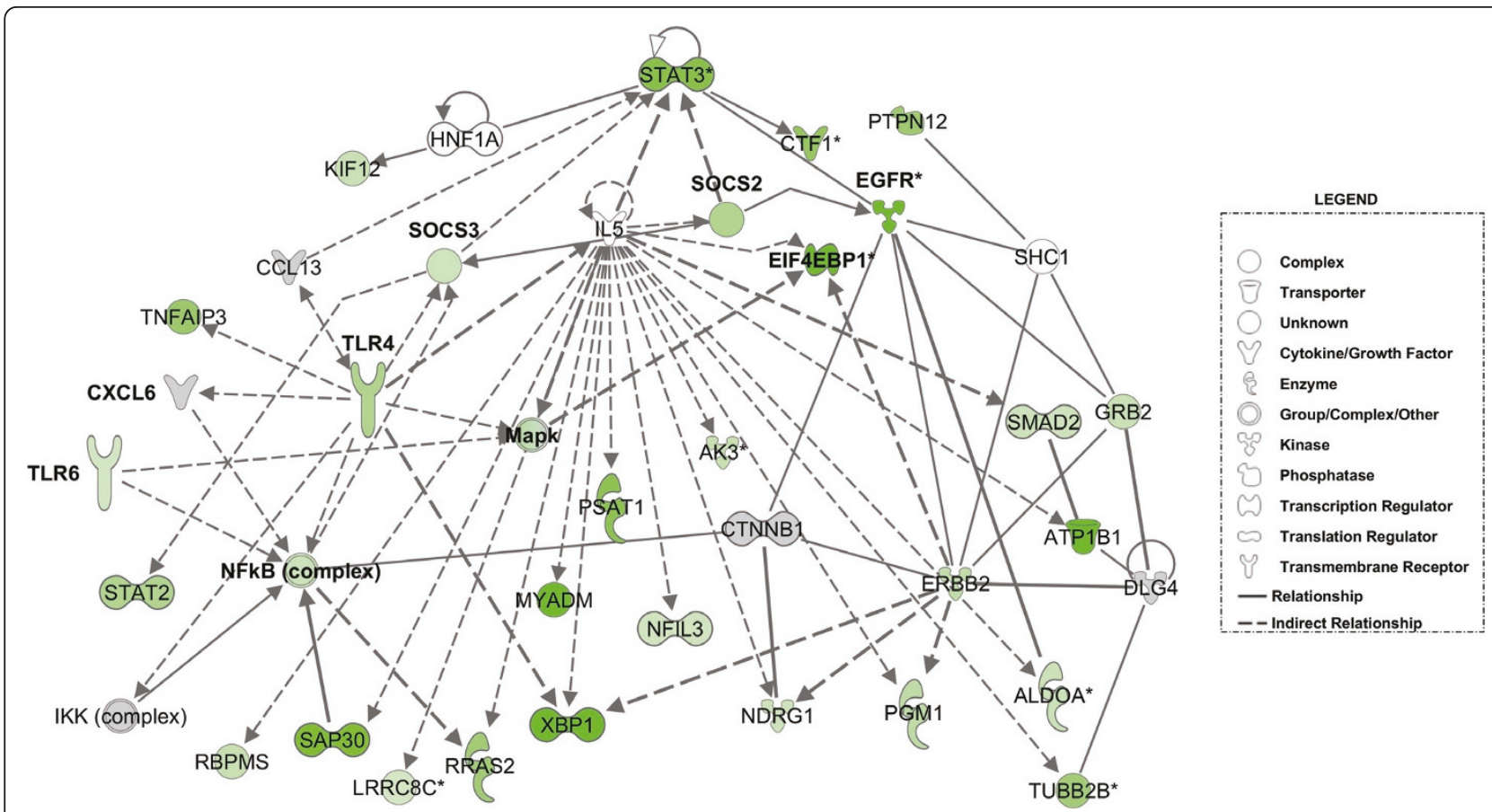

Figure 6 A network of down-regulated genes in quiescent cells after E1A expression. The intensity of the node color (green) indicates the expression level or degree of down-regulation. The genes displayed either in grey or without color were not found in our list of significantly regulated genes. TLRA, NFKB (complex) and ERBB2 are important to the formation of this network.

E2F1 (reverse), 5'-CCCTTGCTTCAG AGAACAGG-3'; MCM7 (forward), 5'-CGGAGATCTA TGGACATGAA3', MCM7(reverse),5'-CATAAGCAGATGTGGATGT-3'; GAPDH (forward), 5'AAGGCCGAGAATGGGAAG-3', GAPDH (reverse), 5'-CTAAGCAGTT GGTGGTGCAG3'; UNG (forward), 5'-TAATCAAGCTCACGGGCTCT-3', UNG (reverse), 5'-GCCAGGATGAACAAAACCAT-3'; PLK1 (forward), 5'-AATAGGG GATTTTGGCTTGG-3', PLK1(reverse), 5'-AATGGACCACACATCCACCT-3'; Skp2 (forward), 5'-GAAACGAGTCAAGGGCAAAG-3', Skp2 (reverse) 5'-AAGGAGCA GCTCATCTGGAA-3'. QRT-PCR analysis was performed by using an iCycler $\mathrm{iQ}^{\mathrm{TM}}$ machine (Bio-Rad), and relative changes were calculated by the $\Delta \Delta \mathrm{Ct}$ method using GAPDH or the $18 \mathrm{~S}$ ribosomal RNA as a reference control gene.

\section{Statistical Analysis}

\section{Microarray Chips Pre-processing}

Output files generated by the Illumina's BeadStudio application containing raw intensities were initially corrected for global normalization, variance-stabilization, and normality in order to identify and remove sources of systematic variation due to experimental artefacts (non-random or technical) and to subsequently perform proper statistical inferences. For this purpose, we used a Variance-Stabilizing Transformation (VST) which is a generalized-log(.) transformation (also known as arcsinh (.)) [30], ensuring that the variance is approximately independent of the mean intensity, followed by a Robust Spline Normalization (RSN) algorithm to the previously variance-stabilized data. The latter procedure is designed to combine the features of quantile and loess normalization. Details of the algorithm are published elsewhere [30]. The raw and preprocessed microarray datasets have been made MIAME-compliant and deposited in the Gene Expression Omnibus (GEO) under accession number GSE28420. These datasets can be accessed and downloaded at: http://www.ncbi.nlm.nih.gov/geo/query/ acc.cgi? acc $=$ GSE28420. To address the usual pre-processing issues upstream of the statistical analysis per se, we employed numerous implementations and algorithms available from the $\mathrm{R}$ project, which is a programming language and platform for statistical computing and visualization (http://www.r-project.org/), and from the Bioconductor project [31] (http://www.bioconductor. $\operatorname{org} /$ ), which is a bioinformatics platform (also in the $\mathrm{R}$ language). Both platforms, which offer powerful statistics, bioinformatics, and visualization tools are freely available to academic users from the Comprehensive $\mathrm{R}$ Archive Network (CRAN) consortium (http://cran.r-project.org/). Specifically, for exploratory data analysis, preprocessing and quality control, we used the $\mathrm{R}$ package 


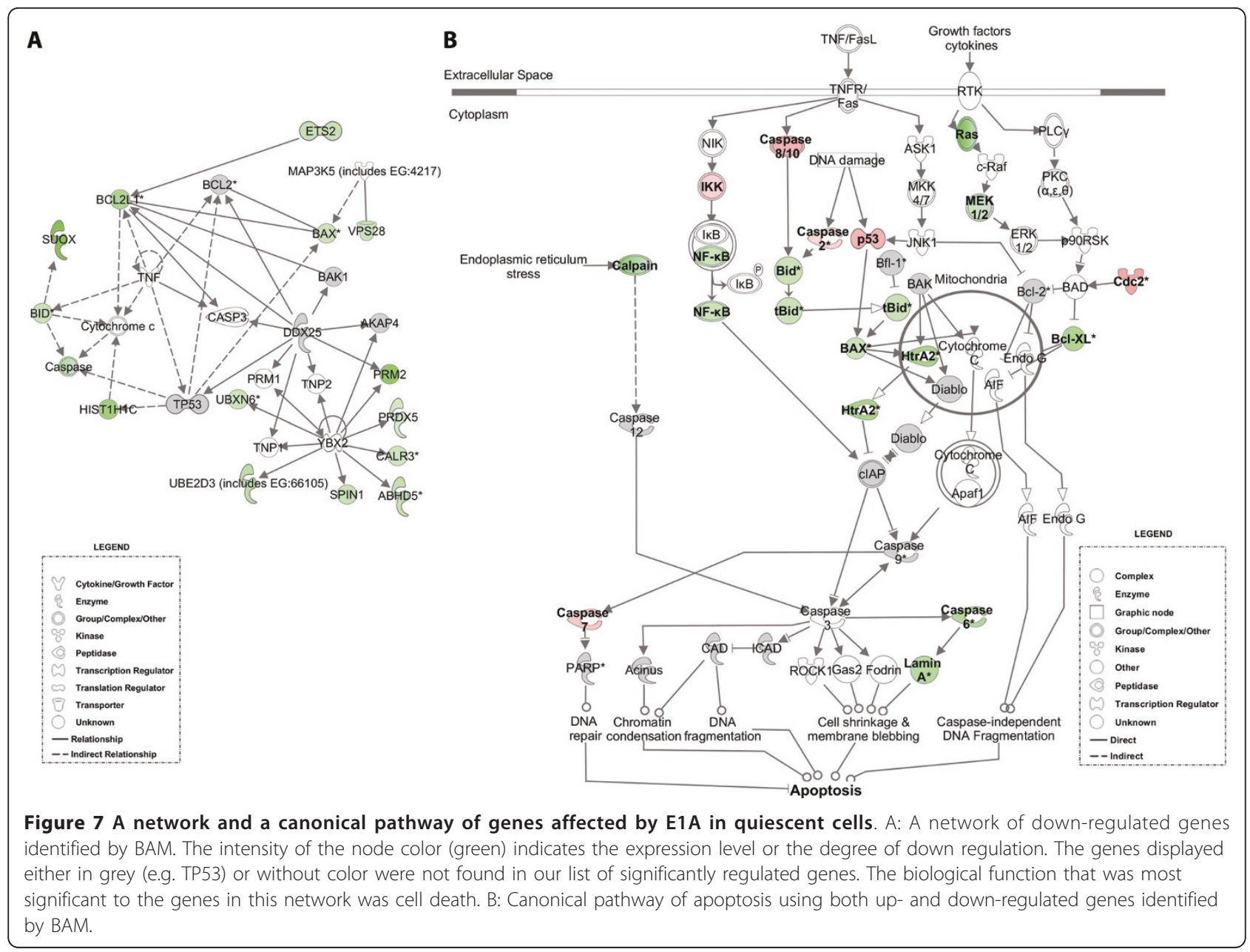

"Lumi" [32], which is especially designed to process the Illumina microarray data.

\section{Pre-filtering and Bayesian Model Selection}

The initial dataset contained 24,613 probes and ESTs uniquely identified by TargetIDs, and from which lists of pre-filtered probes satisfying certain (or a combination of) criterion were drawn: Before the Bayesian model selection analysis was used, a 'Present' or 'Absent' detection call was computed for each probe, similarly to the one described in [33]. The threshold for the 'Present' call $p$-value was set to $p=0.01$. We selected those probes that have a 'Present' detection call $(p=0.01)$ in at least two out of 3 replicates and in at least one experimental group, leaving 10,632 probes. These probes were then used in a Bayesian model selection algorithm [15-17] to determine which of those were differentially expressed. The algorithm is currently implemented as stand-alone Java-based software freely available to academic users: Bayesian Analysis of Microarray (BAM) (http://www.bamarray.com). We report and use the Group Mean Difference and Zcut values in plots and tables. The Group Mean Difference value is simply calculated as the difference between the group means on the transformed scale (i.e. variance stabilization step as described in pre-processing section above). The Zcut value can be seen as Bayesian test statistic equal to the regular $Z$-test statistic from an ANOVA model for comparing two group means, and modulated by a real-valued coefficient bounded by 0 and 1 , called a shrinkage factor. The closer the shrinkage factor is to 0 , the more shrinkage there is, while the closer it is to 1 , the closer Zcut is to the frequentist value [15-17].

\section{Gene Annotations and Gene Ontologies (GO)}

The Illumina microarray uses by default the TargetID identifiers for each 50mer sequence probe, which are not necessarily consistent among different versions of arrays. Therefore, we used the Nucleotide Universal Identifier (NuID) [34], which uniquely matches a 50mer oligonucleotide sequence and contains an error checking and selfidentification code. By using the NuID, designers were able to build one annotation database for different versions of the human (or other species) chips [34]. Moreover, the $\mathrm{NuID}$ can be directly converted to the probe sequence, 
and used to get the most updated RefSeq matches and annotations. For functional annotations and functional analyses, we used the BIOCONDUCTOR platform (http:// www.bioconductor.org/), which is a bioinformatics project (also in the $\mathrm{R}$ language). For the functional annotations and Gene Ontologies, we used the bioinformatics packages "annotate" and "GOstats". To perform enrichment analyses of GO categories among the specified gene symbol sets, we computed hypergeometric $p$-values for over or under-representation of each GO term in the specified category. The category enrichment was computed using a hypergeometric test statistic, based on the observed number of gene symbols representing that category and the expected number one would get by chance alone (i.e. under the null hypothesis of a random phenomenon).

\section{Pathways and Network Analysis Canonical Pathways}

Probe identifiers and Zcut values outputted from the Bayesian model of differentially regulated genes were up-loaded onto the Ingenuity Pathway Analysis (IPA version 8.5) website (http://www.ingenuity.com/ - Ingenuity Systems, Inc., Mountain View, CA) for canonical pathways and network analyses. Under the "General Settings" of the analysis, we first defined the "Reference Set" for our statistical tests (see details below). We used the set of 10,632 pre-filtered probes (see filtering section above) as a "User Dataset" since only those probes (genes) that were pre-selected had a chance of being monitored for enrichment and/or selected as differentially regulated (i.e. included in the test for differential expression). Therefore, this restricted set of genes only should be considered as "Reference Set" $[35,36]$, which is to be distinguished from the total set of 24,613 uniquely identified probes and ESTs present in the MouseRef- 8 v1.1, or from the Ingenuity Knowledge Database. For "Network Analysis", we included Direct and Indirect relationships as well as endogenous chemicals. The IPA "Data Source" was setup to "All knowledge databases", and to avoid analyzing noise in the dataset, we considered only those molecules/genes and/or relationships consistent with our experimental design, that is, "Species" was limited to "Mouse" and "Tissues/Cell Lines" to "Organ Systems" and "Uncategorized Cell Lines". The pathway analysis was performed on each individual set of induced and repressed molecules/genes separately to facilitate interpretations, as well as on the combined set of up/ down-regulated molecules/genes to identify pathways in which a significant number of molecules/genes could be up- or down-regulated altogether. This left 385 up/downregulated molecules/genes that were eligible for generating networks, and 1033 for functional and canonical pathways analysis. Moreover, of the down-regulated molecules/ genes, 139 and 441 were eligible for generating networks and functional and canonical pathways, respectively, while
246 and 591 of the up-regulated molecules/genes were respectfully eligible for generating these categories as well. Significance of each individual pathway intrinsic to our list of differentially expressed genes was measured in IPA in two ways: (i) by a ratio (percentage) of those genes found in a given pathway of our list to all those constitutive of the corresponding canonical pathway; (ii) and by Fisher exact test 'right-tailed' $p$-value for the probability under the null hypothesis that there is no association between those set of genes found in a given pathway of our list with all those constitutive of the corresponding canonical pathway. The smaller the $p$-value, the less likely it is that the association is random and the more significant the association. The difference between the reported ratio and the $p$-values is a matter of percentage vs. probability. The ratio gives the amount of association; while the $p$-value gives a significance or confidence of association. Whenever a multiple-testing correction was required to assess significance of pathway enrichment (e.g. in a hypothesis generating query), we reported adjusted $p$-values using the Benjamini-Hochberg (BH) method [37].

\section{Network Analysis}

Differentially expressed genes were mapped in IPA to the global molecular network that was developed from information in the Ingenuity Knowledge Base. Networks for these genes were algorithmically generated based on their connectivity. A score equal to the negative log of the $p$ value of the right-tailed Fisher's exact test was assigned for each network. This score takes into account the number of eligible genes in our dataset and the size of the network to calculate the fit between each network and the genes in the dataset.

\section{Additional material}

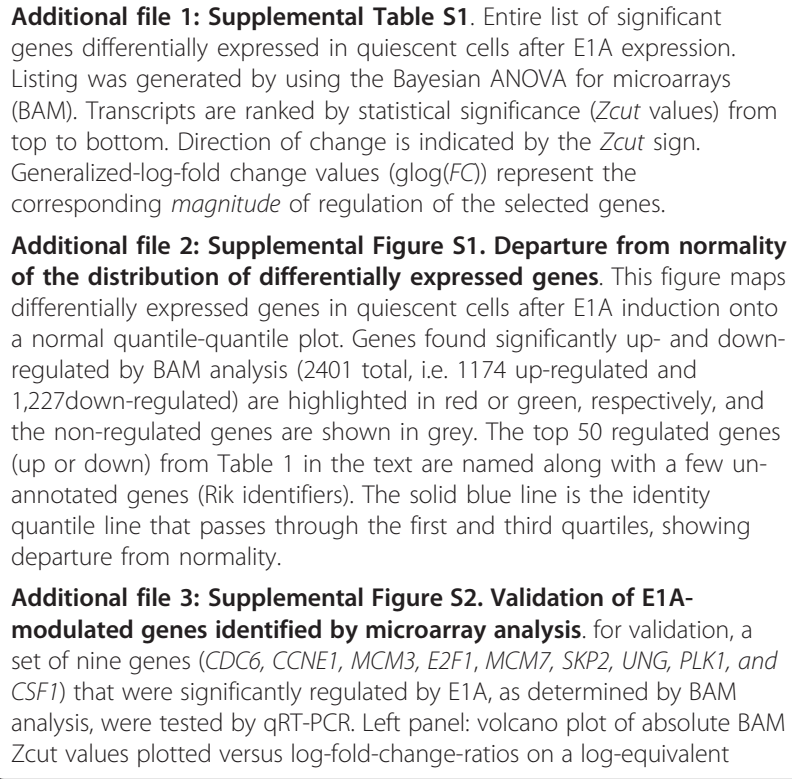

Additional file 2: Supplemental Figure S1. Departure from normality of the distribution of differentially expressed genes. This figure maps differentially expressed genes in quiescent cells after E1A induction onto a normal quantile-quantile plot. Genes found significantly up- and downregulated by BAM analysis (2401 total, i.e. 1174 up-regulated and 1,227down-regulated) are highlighted in red or green, respectively, and the non-regulated genes are shown in grey. The top 50 regulated genes (up or down) from Table 1 in the text are named along with a few unannotated genes (Rik identifiers). The solid blue line is the identity quantile line that passes through the first and third quartiles, showing departure from normality.

Additional file 3: Supplemental Figure S2. Validation of E1Amodulated genes identified by microarray analysis. for validation, a set of nine genes (CDC6, CCNE1, MCM3, E2F1, MCM7, SKP2, UNG, PLK1, and (SF1) that were significantly regulated by E1A, as determined by BAM analysis, were tested by qRT-PCR. Left panel: volcano plot of absolute BAM Zcut values plotted versus log-fold-change-ratios on a log-equivalent 
transformed scale, denoted $M=g \log (\mathrm{S} / \mathrm{Q})$, calculated either from microarray normalized-intensities (red) or qRT-PCR intensities (blue). Note that $M$ values are on identical scale for each assay. Right panel: correlation plot of qRT-PCR fold-change versus microarray normalized-intensities foldchange. Dotted black lines represent the regression and identity lines.

Additional file 4: Supplemental Figure S3. The top metabolic functions affected by E1A in quiescent cells. The stacked bar chart displays for each canonical pathway the number of genes that were found significantly up-regulated (red), and down-regulated (green) by Bayesian model selection. The molecules/genes in a given pathway that were not found in our list of significantly regulated genes are termed unchanged (grey) or not overlapping with our dataset (white). The numerical value at the top of each bar represents the total number of genes/molecules in the canonical pathway. The Benjamini-Hochberg $(\mathrm{BH})$ method was used to adjust the right-tailed Fisher's exact $t$-test $p$-values, which measure how significant each pathway is.

Additional file 5: Supplemental Figure S4. Expression of E1A in E1Ainducible BALB/c 3T3 cells after treatment with Dox. Nuclear extracts were prepared from E1A-inducible cells (Clone 13, passages 12, 18 and 31) after treatment with Dox $(100 \mathrm{ng} / \mathrm{ml})$ for $6 \mathrm{~h}$. Extracts were then subjected to western blot analysis using M73, an antibody specific for E1A $(4,5)$. The membrane was also probed with anti-GAPDH to monitor for equal loading of the extracts.

\section{Acknowledgements}

Supporting information is available on line. We thank David Samols for careful reading of the manuscript. The project described was supported in part by grants from the NIH R01-GM54014 to MLH. JED was partially supported by the Case Comprehensive Cancer Center (NIH P30-CA043703).

\section{Author details}

${ }^{1}$ Center for Proteomics and Bioinformatics, Case Western Reserve University, Cleveland, Ohio 44106, USA. ${ }^{2}$ Department of Biochemistry, School of Medicine, Case Western Reserve University, Cleveland, Ohio 44106, USA. ${ }^{3}$ Division of Cell Biology \& Physiology, Indian Institute of Chemical Biology, West Bengal, India. ${ }^{4}$ The Case Comprehensive Cancer Center, Case Western Reserve University, Cleveland, Ohio 44106, USA.

\section{Authors' contributions}

$J-E D, K Z$, JS, and MLH conceived and designed the experiments. OY, LC, CN, $M G$, and JB performed the experiments. J-ED and MLH analyzed the data. JED and MLH wrote the paper. All authors have read and approved the final manuscript.

\section{Competing interests}

The authors declare that they have no competing interests.

Received: 17 January 2011 Accepted: 26 May 2011

Published: 26 May 2011

\section{References}

1. Miller DL, Myers $\mathrm{CL}$, Rickards B, Coller HA, Flint SJ: Adenovirus type 5 exerts genome-wide control over cellular programs governing proliferation, quiescence, and survival. Genome Biol 2007, 8:R58.

2. Berk AJ: Recent lessons in gene expression, cell cycle control, and cell biology from adenovirus. Oncogene 2005, 24:7673-7685.

3. Spindler KR, Eng CY, Berk AJ: An adenovirus early region 1A protein is required for maximal viral DNA replication in growth-arrested human cells. Journal of Virology 1985, 53:742-750.

4. Ghosh MK, Harter ML: A viral mechanism for remodeling chromatin structure in G0 cells. Mol Cell 2003, 12:255-260.

5. Sha J, Ghosh MK, Zhang K, Harter ML: E1A interacts with two opposing transcriptional pathways to induce quiescent cells into $S$ phase. J Virol 2010, 84:4050-4059.

6. Chattopadhyay D, Ghosh MK, Mal A, Harter ML: Inactivation of p21 by E1A leads to the induction of apoptosis in DNA-damaged cells. J Virol 2001, 75:9844-9856.
7. Mal A, Poon RYC, Howe PH, Toyoshima H, Hunter T, Harter ML: The E1A oncoprotein disables the CDK inhibitor p27 Kip1 in TGF-á treated cells. Nature 1996, 380:262-265.

8. Frolov MV, Dyson NJ: Molecular mechanisms of E2F-dependent activation and pRB-mediated repression. J Cell Sci 2004, 117:2173-2181.

9. Rayman JB, Takahashi Y, Indjeian VB, Dannenberg JH, Catchpole S, Watson RJ, te Riele H, Dynlacht BD: E2F meditates cell cycle-dependent transrciptional repression in vivo by recruitment of an $\mathrm{HDAC} 1 / \mathrm{mSin} 3 \mathrm{~B}$ corepressor complex. Genes \& Dev 2002, 16:933-947.

10. Takahashi Y, Rayman JB, Dynlacht BD: Analysis of promoter binding by the E2F and pRB families in vivo: distinct E2F proteins mediate activation and repression. Genes Dev 2000, 14:804-816.

11. Cam H, Balciunaite E, Blais A, Spektor A, Scarpulla RC, Young R, Kluger $Y$, Dynlacht BD: A common set of gene regulatory networks links metabolism and growth inhibition. Mol Cell 2004, 16:399-411.

12. Xu X, Bieda M, Jin VX, Rabinovich A, Oberley MJ, Green R, Farnham PJ: A comprehensive ChIP-chip analysis of E2F1, E2F4, and E2F6 in normal and tumor cells reveals interchangeable roles of E2F family members. Genome Res 2007, 17:1550-1561.

13. Stacey DW, Dobrowolski SF, Piotrkowski A, Harter ML: The adenovirus E1A protein overrides the requirement for cellular ras in initiating DNA synthesis. EMBO J 1994, 13:6107-6114.

14. Genovese C, Wasserman L: Operating characteristics and extensions of the false discovery rate procedure. J R Statist Soc 2002, 64:499-517

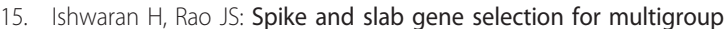
microarray data. J Amer Stat Assoc 2005, 100:764-780.

16. Ishwaran $H$, Rao JS: Spike and slab variable selection: frequentist and Bayesian strategies. The Annals of Statistics 2005, 33:730-773.

17. Ishwaran H, Rao JS: Detecting differentially expressed genes in microarrays using Bayesian model selection. J Amer Stat Assoc 2003, 98:438-455.

18. Ferrari R, Pellegrini M, Horwitz GA, Xie W, Berk AJ, Kurdistani SK: Epigenetic reprogramming by adenovirus e1a. Science 2008, 321:1086-1088.

19. Branzei $D$, Foiani M: Maintaining genome stability at the replication fork. Nat Rev Mol Cell Biol 2010, 11:208-219.

20. Ren B, Cam H, Takahashi Y, Volkert T, Terragni J, Young RA, Dynlacht BD E2F integrates cell cycle progression with DNA repair, replication, and G (2)/M checkpoints. Genes Dev 2002, 16:245-256.

21. Bielas JH, Heddle JA: Quiescent murine cells lack global genomic repair but are proficient in transcription-coupled repair. DNA Repair (Amst) 2004, 3:711-717

22. Nouspikel T: DNA repair in differentiated cells: some new answers to old questions. Neuroscience 2007, 145:1213-1221.

23. Cerezo A, Guadamillas MC, Goetz JG, Sanchez-Perales S, Klein E, Assoian RK, del Pozo MA: The absence of caveolin-1 increases proliferation and anchorage-independent growth by a Rac-dependent, Erk-independent mechanism. Mol Cell Biol 2009, 29:5046-5059.

24. Tsao CC, Geisen C, Abraham RT: Interaction between human MCM7 and Rad17 proteins is required for replication checkpoint signaling. EMBO J 2004, 23:4660-4669.

25. Hublitz $P$, Albert M, Peters $A H$ : Mechanisms of transcriptional repression by histone lysine methylation. Int J Dev Biol 2009, 53:335-354.

26. Hutchens MA, Luker KE, Sonstein J, Nunez G, Curtis JL, Luker GD: Protective effect of Toll-like receptor 4 in pulmonary vaccinia infection. PLoS Pathog 2008, 4:e1000153.

27. Wold WS, Doronin K, Toth K, Kuppuswamy M, Lichtenstein DL, Tollefson AE: Immune responses to adenoviruses: viral evasion mechanisms and their implications for the clinic. Curr Opin Immunol 1999, 11:380-386.

28. Roulston A, Marcellus RC, Branton PE: Viruses and apoptosis. Annu Rev Microbiol 1999, 53:577-628.

29. Mal A, Chattopadhyay D, Ghosh MK, Poon RYC, Hunter T, Harter ML: p21 and retinoblastoma protein control the absence of DNA replication in terminally differentiated muscle cells. J Cell Biol 2000, 149:281-292.

30. Lin SM, Du P, Huber W, Kibbe WA: Model-based variance-stabilizing transformation for Illumina microarray data. Nucleic acids research 2008, 36:e11.

31. Gentleman RC, Carey VJ, Bates DM, Bolstad B, Dettling M, Dudoit S, Ellis B, Gautier L, Ge Y, Gentry J, et al: Bioconductor: open software development for computational biology and bioinformatics. Genome biology 2004, 5 R80.

32. Du P, Kibbe WA, Lin SM: lumi: a pipeline for processing Illumina microarray. Bioinformatics (Oxford, England) 2008, 24:1547-1548. 
33. Liu WM, Mei R, Di X, Ryder TB, Hubbell E, Dee S, Webster TA, Harrington CA, Ho MH, Baid J, et al: Analysis of high density expression microarrays with signed-rank call algorithms. Bioinformatics (Oxford, England) 2002, 18:1593-1599.

34. Du P, Kibbe WA, Lin SM: nulD: a universal naming scheme of oligonucleotides for illumina, affymetrix, and other microarrays. Biology direct 2007, 2:16

35. Rhee SY, Wood V, Dolinski K, Draghici S: Use and misuse of the gene ontology annotations. Nat Rev Genet 2008, 9:509-515.

36. Khatri P, Draghici S: Ontological analysis of gene expression data: current tools, limitations, and open problems. Bioinformatics (Oxford, England) 2005, 21:3587-3595.

37. Benjamini $Y$, Hochberg $Y$ : Controlling the false discovery rate: a practical and powerful approach to multiple testing. J R Statist Soc 1995, 57:289-300.

\section{doi:10.1186/1756-0500-4-160}

Cite this article as: Dazard et al:: The dynamics of E1A in regulating networks and canonical pathways in quiescent cells. BMC Research Notes 2011 4:160.

\section{Submit your next manuscript to BioMed Central} and take full advantage of:

- Convenient online submission

- Thorough peer review

- No space constraints or color figure charges

- Immediate publication on acceptance

- Inclusion in PubMed, CAS, Scopus and Google Scholar

- Research which is freely available for redistribution 